\title{
Verbrennungsmedizin heute
}

$\mathrm{D}$ ie schwere, das heißt tiefe und ausgedehnte Brandverletzung bedeutet für den Organismus ein maximales Trauma, wahrscheinlich das schwerste mögliche Trauma überhaupt. Noch Mitte des vorigen Jahrhunderts endeten Brandverletzungen von über $40 \%$ der Körperoberfläche in der Regel mit dem Tod des Patienten. Trotz aller Fortschritte bei der Behandlung Brandverletzter sind jedoch auch weiterhin das Ausmaß und die Tiefe der Verbrennung, das Alter der Betroffenen oder ein eventuelles Inhalationstrauma die entscheidenden Faktoren, welche die Prognose des schwer verbrannten Patienten bestimmen. Die Rolle vorbestehender Begleiterkrankungen ist erstaunlicherweise in der Literatur nur für die Altersgruppe von mehr als 60 Jahren durch mehrere Untersuchungen belegt.

Die Primärbehandlung schwer brandverletzter Patienten stützt sich heute im Wesentlichen auf drei Säulen. Dazu zählt zum einen die Behandlung des Verbrennungsschocks mit der Stabilisierung der Vitalfunktionen und der intensivmedizinischen Betreuung, die Behandlung der Verbrennungswunde inklusive chirurgischem Debridement und die Deckung mit autologer Spalthaut und zum anderen die primäre Rehabilitation des Patienten.

Gerade in der Anfangsphase der Behandlung lassen sich durch die Beachtung einfacher Grundregeln Fehler vermeiden, die später in vital bedrohlichen Organfunktionsstörungen enden können. Erste wichtige therapeutische Maßnahme ist schon die richtige Zuweisung der Patienten durch den erstversorgenden Notarzt, der die Überweisungskriterien in ein Schwerbrandverletztenzentrum kennen muss, um so eine optimale Behandlung sicherzustellen.

Deutliche Verbesserungen konnten bei der initialen Primärtherapie, der chirurgischen Behandlungsstrategie und der Intensivtherapie inklusive der Ernährungs- und Antibiotikatherapie erzielt werden. Mit diesen Verbesserungen haben sich auch die Therapieziele verschoben. Heute strebt man, nach primärer Stabilisierung des Patienten, eine frühe Deckung der operationspflichtigen verbrannten Areale mit autologer Spalthaut an, um eine lokale oder generalisierte Infektion mit vitaler Bedrohung des Patienten zu verhindern. Dies ermöglicht eine frühe Rehabilitation des Patienten und vermindert die Dauer des Krankenhausaufenthalts sowie die Behandlungskosten.
Die apparative und personelle Ausstattung zur Behandlung schwerbrandverletzter Patienten wie auch eine ausreichende Erfahrung auf ärztlicher und pflegerischer Seite ist nur in spezialisierten Zentren gegeben. Ohne diese Voraussetzungen ist eine moderne erfolgversprechende Behandlung nicht möglich, übersteigt doch der personelle, räumliche, apparative, organisatorische und finanzielle Aufwand den einer normalen Intensivstation um das Vier- bis Fünffache.

Die Betreuung Schwerbrandverletzter erfordert ein hohes Maß an körperlichem, geistigem und seelischem Einsatz nicht nur der betreuenden Ärzte, sondern auch der Pflegekräfte, der Physio- und Ergotherapeuten und der Psychologen. Es dauert Wochen bis ein Brandverletzter aus dem Krankenhaus in die Frührehabilitation entlassen werden kann, die Rückkehr an den Arbeitsplatz ist oft ungewiss. Trotz optimaler Therapie verbleiben Dauerschäden und sichtbare Spuren bis hin zur Entstellung. Narbenkontrakturen zwingen gelegentlich auch Jahre nach dem eigentlichen Brandunfall noch zu wiederherstellenden Operationen.

Diese Schwerpunktausgabe des klinikarzt soll Sie auf die besondere Problematik der Brandverletzung aufmerksam machen und Ihnen einen Überblick über die Grundsätze der Behandlung verschaffen. Anhand spezieller Beispiele dokumentieren die Autoren, wie wichtig eine adäquate Betreuung in ausgewiesenen Zentren ist und welche Vorteile diese für Patienten und Kostenträger hat.

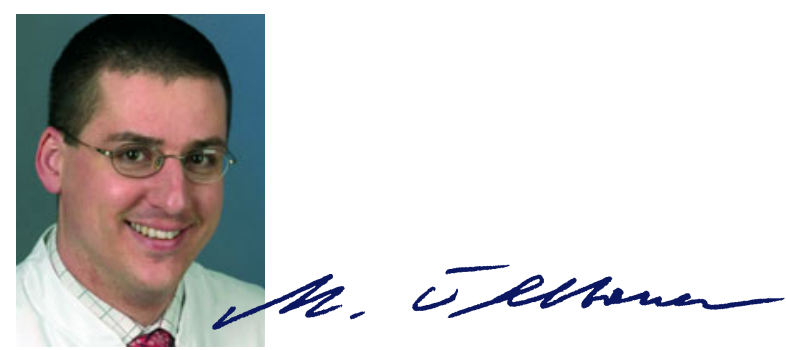

Dr. Markus Öhlbauer, Ludwigshafen Gasteditor 